\title{
THE MAXIMAL KINEMATICAL INVARIANCE GROUP OF FLUID DYNAMICS AND EXPLOSION-IMPLOSION DUALITY
}

\author{
L. O'Raifeartaigh ${ }^{(a)}$ and V. V. Sreedhar ${ }^{(b)}$ \\ School of Theoretical Physics \\ Dublin Institute for Advanced Studies \\ 10, Burlington Road \\ Dublin 4, Ireland
}

\begin{abstract}
It has recently been found that supernova explosions can be simulated in the laboratory by implosions induced in a plasma by intense lasers. A theoretical explanation is that the inversion transformation, $(\Sigma: t \rightarrow-1 / t, \mathbf{x} \rightarrow \mathbf{x} / t)$, leaves the Euler equations of fluid dynamics, with standard polytropic exponent, invariant. This implies that the kinematical invariance group of the Euler equations is larger than the Galilei group. In this paper we determine, in a systematic manner, the maximal invariance group $\mathcal{G}$ of general fluid dynamics and show that it is a semi-direct product $\mathcal{G}=S L(2, R) \wedge G$, where the $S L(2, R)$ group contains the time-translations, dilations and the inversion $\Sigma$, and $G$ is the static (nine-parameter) Galilei group. A subtle aspect of the inclusion of viscosity fields is discussed and it is shown that the Navier-Stokes assumption of constant viscosity breaks the $S L(2, R)$ group to a two-parameter group of time translations and dilations in a tensorial way. The 12-parameter group $\mathcal{G}$ is also known to be the maximal invariance group of the free Schrödinger equation. It originates in the free Hamilton-Jacobi equation which is central to both fluid dynamics and the Schrödinger equation.
\end{abstract}

DIAS-STP-00-16
(a) Deceased
(b) sreedhar@stp.dias.ie 


\section{Introduction}

Considerable efforts are being devoted at present in centres such as the National Ignition Facility at the Lawrence Livermore Laboratory, U.S.A., and the Laser MegaJoule in Bordeaux, France, to simulate astrophysical systems in the laboratory. The motivation for this programme comes from observational evidence [1] that the dynamics of the mixing of gases during a supernova explosion [2] is very similar to the turbulent splashing and mixing of a plasma as a fusion capsule is bombarded by high intensity laser beams [3]. Since the former physical situation deals with an explosion while the latter concerns an implosion, and because the time and length scales involved in the two cases are drastically different, it was a challenging task to produce a theoretical explanation for the observed similarity.

In a recent paper [4], Drury and Mendonça produced such an explanation by showing that the Euler equations of fluid dynamics which govern both the systems are left invariant by an inversion transformation $\Sigma: t \rightarrow-1 / t, \mathbf{x} \rightarrow \mathbf{x} / t$. The discovery of this transformation was inspired by similar transformations of the form $\mathbf{x} \rightarrow f(t) \mathbf{x}$ that are used by cosmologists to factor out an arbitrary uniform expansion or contraction of the system [5]. As is well-known, the Euler equations of fluid dynamics are invariant under the Galilei group [6]. The results of [4] show that the maximal kinematical ${ }^{1}$ invariance group of fluid dynamics $\mathcal{G}$ is larger than the Galilei group [7]. In this paper we determine, in a systematic manner, the structure of $\mathcal{G}$ for general fluid dynamics. We also discuss subtleties associated with the inclusion of viscosity fields and show that the Navier-Stokes assumption of constant viscosity leads to a reduction of $\mathcal{G}$ in a tensorial manner.

1 Meaning transformations involving only space and time coordinates as opposed to those involving internal or gauge degrees of freedom. 
It will be shown that $\mathcal{G}$ is of the form

$$
\mathcal{G}=S L(2, R) \wedge G
$$

where $G$ is the connected, static Galilei group which induces the transformations

$$
\mathbf{x} \rightarrow R \mathbf{x}+\mathbf{a}+\mathbf{v} t, \quad t \rightarrow t
$$

and $S L(2, R)$ is the group

$$
t \rightarrow \frac{\alpha t+\beta}{\gamma t+\delta}, \quad \mathbf{x} \quad \rightarrow \quad \frac{\mathbf{x}}{\gamma t+\delta} ; \quad \alpha \delta-\beta \gamma=1
$$

which includes parity, time translations, dilations and the inversion $\Sigma$.

$\mathcal{G}$ is isomorphic to the Niederer group, which is the maximal invariance group of the free Schrödinger equation [8]. The reason for this isomorphism is that the kinetic part of the fluid-dynamic Lagrangian (see (17)) is of the free Hamilton-Jacobi form, which is the classical limit of the Schrödinger operator.

\section{The Strategy of Proof}

The general fluid dynamic equations in $n$-dimensional space are [9]

$$
\begin{gathered}
D \rho=-\rho \nabla \cdot \mathbf{u} \\
\rho D \mathbf{u}=-\nabla p+\mathbf{V} \\
D \epsilon=-(\epsilon+p) \nabla \cdot \mathbf{u}
\end{gathered}
$$

where the convective derivative $D$ and the viscosity terms $\mathbf{V}$ are defined by

$$
D=\frac{\partial}{\partial t}+\mathbf{u} \cdot \nabla \quad \text { and } \quad V_{i}=\nabla_{j}\left(\eta\left(\nabla_{j} u_{i}+\nabla_{i} u_{j}-\frac{2}{n} \delta_{i j} \nabla_{k} u_{k}\right)\right)+\nabla_{i}\left(\zeta \nabla_{k} u_{k}\right)
$$

respectively. In the above equations $\rho, \mathbf{u}, p$ and $\epsilon$ stand for the density, the velocity vector field, the pressure, and the energy density of the fluid respectively and $\eta, \zeta$ are 
the shear and bulk viscosity fields. The above differential equations are augmented by an algebraic condition called the polytropic equation of state which relates the pressure to the energy density thus:

$$
p=\left(\gamma_{o}-1\right) \epsilon \quad \Rightarrow \quad p+\epsilon=\gamma_{o} \epsilon
$$

$\gamma_{o}$ being a constant called the polytropic exponent. This equation may be used to eliminate $p$ from (4). Further, by making the substitution,

$$
\epsilon=\chi \rho^{\gamma_{o}}
$$

the original set of equations (4) reduces to

$$
\begin{gathered}
D \rho=-\rho \nabla \cdot \mathbf{u} \\
\rho D \mathbf{u}=-\left(\gamma_{o}-1\right) \nabla\left(\chi \rho^{\gamma_{o}}\right)+\mathbf{V} \\
D \chi=0
\end{gathered}
$$

To find the maximal invariance group of these equations we first note that, for any system (such as the above) for which there is no feedback from the fields to the space-time transformations, the maximal invariance group $\mathcal{G}$ of the general class of configurations is also an invariance group for any special sub-class of configurations; though not necessarily a maximal one, since a restricted set of configurations could have a larger symmetry group. Formally,

$$
\mathcal{C}_{s} \subset \mathcal{C} \Rightarrow \mathcal{G} \subseteq \mathcal{G}_{s}
$$

where $\mathcal{C}$ denotes general configurations and $\mathcal{C}_{s}$ denotes a special class. Thus a general strategy for finding maximal invariance groups is to first find a (tractable) sub-class of configurations $\mathcal{C}_{s}$ for which the maximal invariance group $\mathcal{G}_{s}$ can be found, and then see what conditions are imposed on $\mathcal{G}_{s}$ by the general configurations. This is the strategy 
we shall adopt; except that in our case we choose a sub-class for which the generalisation to arbitrary configurations imposes no further conditions i.e. $\mathcal{G}_{s} \equiv \mathcal{G}$. The tractable sub-class we choose is obtained by making three simplifications, namely, ignoring the effects of viscosity by tuning the viscosity fields $\eta$ and $\zeta$ to zero, by setting $\chi=1$, and by letting the velocity vector $\mathbf{u}$ be curl-free. The resulting sub-class is the configuration of inviscid, isentropic and irrotational flows which are well-known in fluid dynamics.

\section{The Sub-class of Inviscid, Isentropic, and Irrotational Fluids}

The first simplification we consider is to ignore viscosity effects in which case (8) reduces to

$$
\begin{gathered}
D \rho=-\rho \nabla \cdot \mathbf{u} \\
\rho D \mathbf{u}=-\left(\gamma_{o}-1\right) \nabla\left(\chi \rho^{\gamma_{o}}\right) \\
D \chi=0
\end{gathered}
$$

Second, we note that it is consistent with the field equations to set $\chi=1$, with $\mathbf{u}$ not necessarily curl-free, in which case the equations in (10) reduce to,

$$
D \rho=-\rho \nabla \cdot \mathbf{u} \quad \text { and } \quad D \mathbf{u}=-\gamma_{o}\left(\gamma_{o}-1\right) \rho^{\gamma_{o}-2} \nabla \rho
$$

The reason for making this simplification is that the system becomes a local Lagrangian one. This can be seen by using the standard Clebsch parametrization [10]

$$
\mathbf{u}=-\nabla \phi-\nu \nabla \theta
$$

for the vector-field $\mathbf{u}$, in which case the local Lagrangian density is,

$$
\mathcal{L}=\rho\left[\dot{\phi}+\nu \dot{\theta}-\frac{1}{2}(\nabla \phi+\nu \nabla \theta)^{2}-\rho^{\gamma_{o}-1}\right]
$$

It will be convenient to write this Lagrangian density in the form

$$
\mathcal{L}=\rho\left[\dot{\phi}-\frac{1}{2}(\nabla \phi)^{2}+\nu \mathcal{D} \theta-\frac{\nu^{2}}{2}(\nabla \theta)^{2}-\rho^{\gamma_{o}-1}\right] \quad \text { where } \quad \mathcal{D}=\frac{\partial}{\partial t}-\nabla \phi \cdot \nabla
$$


is the convective derivative $D$ restricted to the curl-free part of $\mathbf{u}$. Clearly $\nu$ is a Lagrange multiplier, and, by varying it, we obtain

$$
\nu=\frac{\mathcal{D} \theta}{(\nabla \theta)^{2}} \quad \text { and } \quad \mathcal{L}=\rho\left[\dot{\phi}-\frac{1}{2}(\nabla \phi)^{2}-\frac{1}{2} \frac{(\mathcal{D} \theta)^{2}}{(\nabla \theta)^{2}}-\rho^{\gamma_{o}-1}\right]
$$

Note that the first equation here is equivalent to $D \theta=0$ since

$$
D \theta=\mathcal{D} \theta-(\nu \nabla \theta) \cdot \nabla \theta=\mathcal{D} \theta-\nu(\nabla \theta)^{2}=(\nabla \theta)^{2}\left(\frac{\mathcal{D} \theta}{(\nabla \theta)^{2}}-\nu\right)
$$

We now proceed to the third and last simplification mentioned.

The third simplification we make is to consider the curl-free case $\mathbf{u}=-\nabla \phi$ or $\nu=\theta=0$. Then the Action corresponding to the Lagrangian density (13) reduces to

$$
S=\int d^{n} x d t \rho\left[\dot{\phi}-\frac{1}{2}(\nabla \phi)^{2}\right]-\rho^{\gamma_{o}}
$$

Note that the quantity in square brackets is just the Hamilton-Jacobi function for a free particle. Following our strategy we now seek the maximal invariance group of the Action (17).

The most general transformation involving the fields, as shown in the Appendix, takes the linear, inhomogeneous form

$$
\xi_{i}=\xi_{i}(x, t), \quad \tau=\tau(x, t), \quad \tilde{\phi}=s(x, t) \phi+\lambda(x, t), \quad \tilde{\rho}=\mu(x, t) \rho+\mu^{\prime}(x, t)
$$

where $s$ is a constant. However, one sees at once that if $\mu^{\prime} \neq 0$ the form invariance of the Action is violated by inhomogeneous terms. Furthermore, since

$$
\frac{\partial}{\partial t}=\frac{\partial \tau}{\partial t} \frac{\partial}{\partial \tau}+\frac{\partial \xi_{i}}{\partial t} \frac{\partial}{\partial \xi_{i}} \quad \text { and } \quad \frac{\partial}{\partial x_{i}}=\frac{\partial \tau}{\partial x_{i}} \frac{\partial}{\partial \tau}+\frac{\partial \xi_{j}}{\partial x_{i}} \frac{\partial}{\partial \xi_{j}}
$$

we see that we obtain a term $(\partial \tilde{\phi} / \partial \tau)^{2}$ unless

$$
\frac{\partial \tau}{\partial x_{i}}=0
$$


This means that the $\partial \phi / \partial t$ term does not pick up an $x$-dependent factor relative to $\partial \phi / \partial \tau$. Finally we note that under the transformations (18), the second term in the Hamilton-Jacobi part of the Action (17) gives

$$
\frac{1}{2} h_{i j} \frac{\partial \phi}{\partial x_{i}} \frac{\partial \phi}{\partial x_{j}} \rightarrow \frac{1}{2} g_{i j} \frac{\partial \phi}{\partial \xi_{i}} \frac{\partial \phi}{\partial \xi_{j}} \quad \text { where } g_{i j}=\frac{\partial \xi_{i}}{\partial x_{k}} \frac{\partial \xi_{j}}{\partial x_{l}} h_{k l} \equiv \Omega(x) g_{i j}^{\prime}(\xi)
$$

where $h_{i j}$ is the flat Euclidean metric. As is well-known, the most general transformations that have the above property are the special conformal transformations with parameter $b$, for which $\Omega=\left(1+2 b \cdot x+b^{2} x^{2}\right)^{2}$, and the Euclidean transformations for which $\Omega=1$. It is easy to see however that, for the Action in (17) to remain invariant, the $\partial \phi / \partial t$ part of the Hamilton-Jacobi function must transform in the same way as the $(\partial \phi / \partial x)^{2}$ part. But since $(20)$ forbids $\partial \phi / \partial t$ to pick up an $x$-dependent factor, the conformal transformations in (21) are not allowed. Thus the possible transformations reduce to

$$
\xi_{i}=M_{i j}(t) x_{j}+w_{i}(t), \quad \tau=\tau(t), \quad \tilde{\phi}=s \phi+\lambda(x, t) \quad \tilde{\rho}=\mu(x, t) \rho
$$

where $s$ is a constant and $M$ is Euclidean i.e. $M=f(t) R(t)$, where $f$ is a scale factor and $R(t)$ is a rotation matrix. In that case

$$
\left(\begin{array}{cc}
\frac{\partial \tau}{\partial t} & \frac{\partial \tau}{\partial x_{j}} \\
\frac{\partial \xi_{i}}{\partial t} & \frac{\partial \xi_{i}}{\partial x_{j}}
\end{array}\right)=\left(\begin{array}{cc}
\dot{\tau} & 0 \\
\dot{\xi}_{i} & M_{i j}
\end{array}\right) \Rightarrow\left(\begin{array}{c}
\dot{\tau} \neq 0 \\
\operatorname{det} M \neq 0
\end{array}\right)
$$

and the Action becomes

$$
\begin{aligned}
S & =\int \frac{d^{n} \xi d \tau}{\operatorname{det} M^{n} \dot{\tau}} \mu^{-1} \tilde{\rho}\left[\left(\dot{\tau} \frac{\partial}{\partial \tau}+\dot{\xi}_{i} \frac{\partial}{\partial \xi_{i}}\right) \frac{(\tilde{\phi}-\lambda)}{s}-\frac{\operatorname{det} M^{2}}{2 s^{2}}\left(\frac{\partial \tilde{\phi}}{\partial \xi_{i}}-\frac{\partial \lambda}{\partial \xi_{i}}\right)^{2}-\left(\mu^{-1} \tilde{\rho}\right)^{\gamma_{o}-1}\right] \\
& =\int \frac{d^{n} \xi d \tau}{s \mu \operatorname{det} M^{n}} \tilde{\rho}\left[\left(\frac{\partial}{\partial \tau}+\frac{\dot{\xi}_{i} \frac{\partial}{\partial \xi_{i}}}{\dot{\tau}}\right)(\tilde{\phi}-\lambda)-\frac{\operatorname{det} M^{2}}{2 s \dot{\tau}}\left(\frac{\partial \tilde{\phi}}{\partial \xi_{i}}-\frac{\partial \lambda}{\partial \xi_{i}}\right)^{2}-\frac{s\left(\mu^{-1}\right)^{\gamma_{o}-1}}{\dot{\tau}} \tilde{\rho}^{\gamma_{o}-1}\right]
\end{aligned}
$$

Invariance then requires that

$$
s \mu(\operatorname{det} M)^{n}=1, \quad(\operatorname{det} M)^{2}=s \dot{\tau}, \quad \text { and } \quad\left(\mu^{-1}\right)^{\gamma_{o}-1}=\frac{\dot{\tau}}{s}
$$


and

$$
\left(\frac{\partial}{\partial \tau}+\frac{\partial \xi_{i}}{\partial \tau} \frac{\partial}{\partial \xi_{i}}\right)(\tilde{\phi}-\lambda)-\frac{1}{2}\left(\frac{\partial \tilde{\phi}}{\partial \xi}-\frac{\partial \lambda}{\partial \xi}\right)^{2}=\frac{\partial \tilde{\phi}}{\partial \tau}-\frac{1}{2}\left(\frac{\partial \tilde{\phi}}{\partial \xi}\right)^{2}
$$

Equations (25) can be readily solved to get

$$
s=1 \Rightarrow(\operatorname{det} M)^{2}=\dot{\tau}, \quad \mu=(\operatorname{det} M)^{-n}, \quad \text { and } \quad \gamma_{o}=1+\frac{2}{n}
$$

The result for $\gamma_{o}$ is in agreement with [4]; in particular in three-dimensional space,

$n=3$, and $\gamma_{o}=\frac{5}{3}$, as expected. Substituting these results in (26), and requiring that it be satisfied for all $\partial \tilde{\phi} / \partial \xi_{i}$, splits it into

$$
\frac{\partial \xi_{i}}{\partial \tau}=-\frac{\partial \lambda}{\partial \xi_{i}} \text { and } \quad \frac{\partial \lambda}{\partial \tau}-\frac{1}{2}\left(\frac{\partial \lambda}{\partial \xi}\right)^{2}=0, \quad \text { where } \quad \dot{\tau}=\operatorname{det} M^{2}
$$

Notice that the second equation is just the free Hamilton-Jacobi equation with Action $\lambda$. The maximal invariance group can now be obtained by solving the above equations for the functions $\xi_{i}$ and $\lambda$. Thus the invariance group $\mathcal{G}$ is determined by the structure of the Hamilton-Jacobi equation for a free particle. However, we will now show, even before solving these equations, that the group of invariance remains the same when the simplifications made in this section are relaxed and we return to the case of the general fluid configurations.

\section{Proof of Maximality for General Fluid Configurations}

We first note that the first and last equations in (28) are exactly the conditions for the restricted derivative $\mathcal{D}$ to transform covariantly since

$$
\begin{aligned}
\mathcal{D}(x, t, \phi) & =\frac{\partial}{\partial t}-\frac{\partial \phi}{\partial x_{i}} \frac{\partial}{\partial x_{i}}=\left(\dot{\tau} \frac{\partial}{\partial \tau}+\frac{\partial \xi_{i}}{\partial t} \frac{\partial}{\partial \xi_{i}}\right)-\left(\frac{\partial \xi_{j}}{\partial x_{i}} \frac{\partial \xi_{k}}{\partial x_{i}}\right) \frac{\partial \phi}{\partial \xi_{j}} \frac{\partial}{\partial \xi_{k}} \\
& =\left(\dot{\tau} \frac{\partial}{\partial \tau}-\operatorname{det} M^{2} \frac{\partial \tilde{\phi}}{\partial \xi_{i}} \frac{\partial}{\partial \xi_{i}}\right)+\left(\frac{\partial \xi_{i}}{\partial t}+\operatorname{det} M^{2} \frac{\partial \lambda}{\partial \xi_{i}}\right) \frac{\partial}{\partial \xi_{i}}=\operatorname{det} M^{2} \mathcal{D}(\xi, \tau, \tilde{\phi})
\end{aligned}
$$


Let us now relax the assumption that $\mathbf{u}$ is curl-free, with $\chi=1$ still being true. From the Lagrangian density for this case,

$$
\mathcal{L}=\rho\left[\dot{\phi}-\frac{1}{2}(\nabla \phi)^{2}-\frac{1}{2} \frac{(\mathcal{D} \theta)^{2}}{(\nabla \theta)^{2}}-\rho^{\gamma_{o}-1}\right]
$$

and the covariant transformations,

$$
\mathcal{D}(x, t, \phi)=(\operatorname{det} M)^{2} \mathcal{D}(\xi, \tau, \tilde{\phi}) \quad \text { and } \quad \nabla(x)=(\operatorname{det} M) \nabla(\xi)
$$

we see that (30) will be invariant under the given space-time transformations provided only that $\nu$ and $\theta$ are scalars. Thus the case of general $\mathbf{u}$ puts no restrictions on the space-time transformations but determines the transformation properties of the $\nu$ and $\theta$ components of $\mathbf{u}$. In particular we see that the full convective derivative $D$ and $\mathbf{u}$ have the transformation properties

$$
D(x, t, \phi)=(\operatorname{det} M)^{2} D(\xi, \tau, \tilde{\phi}), \quad \text { and } \quad \tilde{\mathbf{u}}=\frac{1}{\operatorname{det} M}(\mathbf{u}-\nabla \lambda)
$$

Note that the inhomogeneous part of the $\mathbf{u}$-transformation comes from the curl-free part of $\mathbf{u}$. Furthermore we see that the $\mathcal{G}$ transformations do not preserve the condition of incompressibility $(\nabla \cdot \mathbf{u}=0)$ and hence we need to consider general, compressible fluid configurations.

All this is within the Lagrangian framework. In order to relax the $\chi=1$ simplification, we need to allow for general fields $\chi$. For this we must go outside the Lagrangian framework and consider the field equations. But that is simple since the field equations with $\chi=1$ are invariant and we see by inspection that the equations for general $\chi$ remain invariant provided that $\chi$ transforms as a scalar. Thus the set of equations in (10) is invariant with respect to the maximal invariance group provided $\nu, \theta$ and $\chi$ transform as scalars. Thus the maximal invariance group for $\nu=\theta=0, \chi=1$ (corresponding to our sub-class without viscosity) is the maximal invariance group for all inviscid configurations. 
Finally we include viscosity effects. It is straightforward to verify that the viscosity terms in (4b) are covariant only if $\nabla \cdot \mathbf{u}=0$ or $\nabla_{i} \zeta=0$. However, as already mentioned, the former condition is not invariant under $\mathcal{G}$ transformations and hence covariance requires that $\zeta$ is a constant in space (although not in time). In this case, the fluid equations (4) would remain invariant if the viscosity fields transform as tensors of rank $n$, where $n$ is the space dimension i.e. $\eta \rightarrow \operatorname{det} M^{n} \eta$ and $\zeta \rightarrow \operatorname{det} M^{n} \zeta$. This in turn implies that the equations would remain invariant under the Navier-Stokes assumption of constant viscosity only if $\operatorname{det} M$ is a constant which breaks the $\mathcal{G}$ symmetry in a tensorial way. Therefore the maximal invariance group of the Navier-Stokes equations contains just the static Galilei group plus dilations and time translations corresponding to $\operatorname{det} M$ being a constant, as will be clear when we solve for the $\mathcal{G}$ transformation functions in the next section.

It is worth mentioning that the breakdown of $\mathcal{G}$ by the Navier-Stokes restriction does not contradict (9) because, in contrast to the other restrictions $\eta=\zeta=0, \chi=1$, $\nabla \times \mathbf{u}=0$ that we have considered, this restriction has a feed-back effect on the nonlinear part of the space-time transformations.

\section{The Solutions for the Transformation Functions}

Returning to the equations in (28), it is first useful to note from the definition of $\xi$ in (22) that

$$
\frac{\partial \xi_{i}}{\partial \tau}=A_{i j} \xi_{j}+W_{i} \text { where } A \equiv M_{\tau} M^{T}=f_{\tau} f^{-1}+R_{\tau} R^{T} \text { and } W=M \frac{\partial}{\partial \tau}\left(M^{-1} w\right)
$$

where the subscript $\tau$ denotes differentiation with respect to $\tau$. Hence the first equation in (28) becomes

$$
\frac{\partial \lambda}{\partial \xi_{i}}=-A_{i j} \xi_{j}-W_{i} \quad \Rightarrow \quad\left(\frac{\partial^{2} \lambda}{\partial \xi_{i} \partial \xi_{j}}=-A_{i j}\right)
$$


Here the equation in the brackets shows that $A$ is a symmetric matrix. But since $R$ is a rotation matrix, $R_{\tau} R^{T}$ is in the Lie algebra of the rotation group and hence is antisymmetric. Thus we have the result $R_{\tau}=0$ and hence $R$ is a constant (rigid) rotation matrix. ${ }^{2}$

It then follows that $\operatorname{det} M=f$ and from (28) and (32) we have

$$
\dot{\tau}=f^{2} \quad \text { and } \quad \tilde{\mathbf{u}}=\frac{1}{f}(\mathbf{u}-\nabla \lambda)
$$

The definitions of $A$ and $W$ then simplify to

$$
A=f_{\tau} f^{-1} \quad \text { and } \quad W_{i}=f \partial_{\tau}\left(\frac{w_{i}}{f}\right)
$$

Integrating (34) with respect to $\xi$ we have

$$
\lambda=-A \frac{\xi^{2}}{2}-W_{i} \xi_{i}-h(\tau)
$$

Substituting this into the second equation in (28) we get

$$
-\frac{(\xi)^{2}}{2} \frac{\partial A}{\partial \tau}-\xi_{i} \frac{\partial W_{i}}{\partial \tau}-\frac{\partial h}{\partial \tau}=\frac{1}{2}(A \xi+W)^{2}
$$

Comparing the coefficients of the powers of $\xi$ breaks this into

$$
\frac{\partial A}{\partial \tau}=-A^{2}, \quad \frac{\partial W_{i}}{\partial \tau}=-A W_{i}=-\frac{\partial_{\tau} f}{f} W_{i}, \quad \frac{\partial h}{\partial \tau}=-\frac{W^{2}}{2}
$$

We can solve the second equation in (39) explicitly to get

$$
W_{i}=\frac{v_{i}}{f} \quad \Rightarrow \quad \partial_{t}\left(\frac{w_{i}}{f}\right)=v_{i} \quad \Rightarrow \quad w_{i}=f(t)\left(v_{i} t+a_{i}\right)
$$

where the $v_{i}$ and $a_{i}$ are constants. It follows that

$$
\xi_{i} \equiv f x_{i}+w_{i}=f(t)\left(x_{i}+a_{i}+v_{i} t\right)
$$

\footnotetext{
${ }^{2}$ With this in mind, we suppress the matrix for convenience, and restore it later.
} 
The third equation in (39) can then be written as

$$
\frac{\partial h}{\partial \tau}=-\frac{v^{2}}{2 f^{2}} \Rightarrow \frac{\partial h}{\partial t}=-\frac{v^{2}}{2} \Rightarrow h=h_{0}-\frac{v^{2}}{2} t
$$

Since the transformations of the fields are given by $\tilde{\rho}=f^{-n} \rho$ and $\tilde{\phi}=\phi+\lambda$, we see that everything is determined by the function $f$ which satisfies

$$
\frac{\partial}{\partial \tau}\left(\frac{\dot{f}}{f^{3}}\right)=-\left(\frac{\dot{f}}{f^{3}}\right)^{2}
$$

Using $\dot{\tau}=f^{2}$, this is easily seen to be equivalent to

$$
\partial_{\tau}^{2} f=0 \quad \text { and } \quad \frac{\dddot{\tau}}{\dot{\tau}}-\frac{3}{2}\left(\frac{\ddot{\tau}}{\dot{\tau}}\right)^{2}=0
$$

The left hand side of the second equation is the Schwarzian derivative of $\tau$ and it follows that the general solution of the above equation is

$$
\tau=\frac{\alpha t+\beta}{\gamma t+\delta} \quad \text { and } \quad f=\frac{1}{\gamma t+\delta} \quad \text { where } \quad \alpha \delta-\beta \gamma=1
$$

Thus the most general transformations of the time coordinate form an $S L(2, R)$ group. The second equation in (35) then reduces to

$$
\tilde{\mathbf{u}}=(\gamma t+\delta) \mathbf{u}-\gamma(\mathbf{x}+\mathbf{a})+\delta \mathbf{v}
$$

From (41) it also follows that

$$
\xi_{i}=\frac{1}{\gamma t+\delta}\left(x_{i}+a_{i}+v_{i} t\right)
$$

Returning to the general case, and using the inversions

$$
\gamma \tau-\alpha=\frac{-1}{\gamma t+\delta} \quad \text { and } \quad \gamma \xi-v=\frac{\gamma(x+a)-\delta v}{\gamma t+\delta}
$$

we have

$$
\lambda=\lambda_{0}-\frac{(\gamma \xi-v)^{2}}{2 \gamma(\gamma \tau-\alpha)}=\lambda_{0}+\frac{[\gamma(x+a)-\delta v]^{2}}{2 \gamma(\gamma t+\delta)} \quad \text { where } \quad \lambda_{0}=-\left(h_{0}+\frac{\delta}{\gamma} \frac{v^{2}}{2}\right)
$$


The case $\operatorname{det} M=f=$ a constant corresponds to letting $\gamma=0$ and from (45) and (47) it is clear that the group of transformations in this case includes the static Galilei group $G$, the dilations, the time translations and parity, but excludes time-reversal. As already mentioned, this is the maximal invariance group of the Navier-Stokes equations. It is also useful to consider the following two special cases.

I. $\beta=\gamma=0, \alpha=1$ : The Connected, Static Galilei Transformations: In this case, we have

$$
g: \quad \tau=t, \quad \xi=R \mathbf{x}+\mathbf{a}+\mathbf{v} t
$$

where we have restored the rotations, and from (49) it follows

$$
\lambda=-h_{0}-\mathbf{v} \cdot(R \mathbf{x}+\mathbf{a})-\frac{v^{2}}{2} t
$$

These equations describe connected, static Galilei transformations which exclude parity and time-reversal.

II. $\mathbf{a}=\mathbf{v}=\mathbf{0}, R=1$ : The Inversion Transformations: In this case, we have

$$
\sigma: \quad \tau=\frac{\alpha t+\beta}{\gamma t+\delta}, \quad \xi=\frac{R \mathbf{x}}{\gamma t+\delta} ; \quad \alpha \delta-\beta \gamma=1
$$

These are the $S L(2, R)$ generalisations of the inversion transformations presented in [4]. For the transformations of the fields we have from (49)

$$
\lambda=\frac{\gamma x^{2}}{2(\gamma t+\delta)}-h_{0}
$$

\section{The Maximal Invariance Group $\mathcal{G}$}

To understand the structure of the group, we study the relationship between the $S L(2, R)$ group and the connected static Galilei group $G$. Let us first consider a conjugation of a $g \in G$ by a $\sigma \in S L(2, R)$. By making three successive transformations of $x$ and $t$ we find that

$$
\sigma^{-1}(\alpha, \beta, \gamma) g(R, \mathbf{a}, \mathbf{v}) \sigma(\alpha, \beta, \gamma)=g\left(R, \mathbf{a}_{\sigma}, \mathbf{v}_{\sigma}\right)
$$


where

$$
\left(\begin{array}{l}
\mathbf{a}_{\sigma} \\
\mathbf{v}_{\sigma}
\end{array}\right)=\left(\begin{array}{ll}
\delta & \beta \\
\gamma & \alpha
\end{array}\right)\left(\begin{array}{l}
\mathbf{a} \\
\mathbf{v}
\end{array}\right)
$$

This shows that $G$ is an invariant sub-group and so the group structure is

$$
\mathcal{G}=S L(2, R) \wedge G
$$

where $\wedge$ denotes semi-direct product with $G$ as invariant subgroup. More precisely, if we recall that $G$ itself takes the form

$$
G=R \wedge(T(\mathbf{a}) \otimes B(\mathbf{v}))
$$

where $T$ and $B$ are the translation and boost groups with parameters $\mathbf{a}$ and $\mathbf{v}$ respectively, then we see that $S L(2, R)$ commutes with $R$ and mixes $T$ and $B$ in the manner shown in $(55)$.

The original inversion $\Sigma$ is the special element of $S L(2, R)$ for which $(\alpha, \beta, \gamma, \delta)=$ $(0,-1,1,0)$. Note that $\Sigma^{2}=P$ where $P$ is the parity. Furthermore if we consider the coset elements $g_{\Sigma}(R, \mathbf{a}, \mathbf{v}) \equiv \Sigma g(R, \mathbf{a}, \mathbf{v})$, where $g \in G$, we have using (54) and the standard property of Galilei transformations viz. $g(R, \mathbf{a}, \mathbf{v}) g\left(R^{\prime}, \mathbf{a}^{\prime}, \mathbf{v}^{\prime}\right)=g\left(R R^{\prime}, R \mathbf{a}^{\prime}+\right.$ $\left.\mathbf{a}, R \mathbf{v}^{\prime}+\mathbf{v}\right)$,

$$
\begin{gathered}
g_{\Sigma}\left(R^{\prime}, \mathbf{a}^{\prime}, \mathbf{v}^{\prime}\right) g_{\Sigma}(R, \mathbf{a}, \mathbf{v})=g_{P}\left(R^{\prime} R, R^{\prime} \mathbf{a}-\mathbf{v}^{\prime}, R^{\prime} \mathbf{v}+\mathbf{a}^{\prime}\right) \\
\Rightarrow \quad g_{\Sigma}^{2}(R, \mathbf{a}, \mathbf{v})=g_{P}\left(R^{2}, R \mathbf{a}-\mathbf{v}, R \mathbf{v}+\mathbf{a}\right)
\end{gathered}
$$

where we have used the obvious notation $g_{P}(R, \mathbf{a}, \mathbf{v})=\operatorname{Pg}(R, \mathbf{a}, \mathbf{v})$. It follows from the above equation that

$$
g_{\Sigma}^{4}(R, \mathbf{a}, \mathbf{v})=g\left(R^{4},\left(R^{2}-1\right)(R \mathbf{a}-\mathbf{v}),\left(R^{2}-1\right)(R \mathbf{v}+\mathbf{a})\right)
$$

Since $R \mathbf{a}-\mathbf{v}$ and $R \mathbf{v}+\mathbf{a}$ are linearly independent, this shows that every connected Galilei transformation is the fourth power of a coset transformation. 


\section{Extension to the Quantum Theory}

If a classical system is described by the Galilei group $G$ in (57) then, in the field theoretic representation, there is a central extension of $G$; namely, the well-known oneparameter mass group whose generator commutes with all the generators of $G$. Since a quantum wavefunction can be thought of as a nonrelativistic field, the corresponding quantum system is described by a central extension of the group of the form

$$
G=R \wedge(T(\mathbf{a}), B(\mathbf{v}))
$$

where the group $(T, B)$ is no longer abelian, but is a Heisenberg-Weyl group of the form

$$
T(\mathbf{a}) B(\mathbf{v})=B(\mathbf{v}) T(\mathbf{a}) e^{i \hbar M \mathbf{a} \cdot \mathbf{v}}
$$

and $M$ is a central constant. It is obvious that this relation is invariant with respect to the transformation (55) of the parameters $\mathbf{a}$ and $\mathbf{v}$ induced by $S L(2, R)$. Thus $\mathcal{G}$ remains a symmetry group of the quantised system.

\section{Connection to the Free Schrödinger Equation}

The fact that $\mathcal{G}$ is a covariance group of the Hamilton-Jacobi function $\partial \phi / \partial t+$ $1 / 2(\nabla \phi)^{2}$ shows that it is an invariance group of a free particle. The above discussion of the quantum extension implies that it is also an invariance group of a quantised free particle. Indeed it was shown in 1972 that $\mathcal{G}$ is the maximal invariance group of the free particle Schrödinger equation [8]. Since the invariance under the Galilei group is well-known, it suffices to verify that the Schrödinger equation

$$
i \hbar \frac{\partial \psi}{\partial t}+\frac{\hbar^{2}}{2 m} \frac{\partial^{2} \psi}{\partial x_{i}^{2}}=0
$$

remains form-invariant under the $\mathcal{G}$. It is easily checked that this is accomplished by the following transformation of the wavefunction

$$
\psi(\mathbf{x}, t) \sim(\gamma \tau-\alpha)^{\frac{n}{2}} e^{-i \lambda} \psi(\xi, \tau) \sim(\gamma t+\delta)^{-\frac{n}{2}} e^{-i \lambda} \psi
$$


where $\lambda$ is defined in (49). Note that at time $\tau=\infty$, i.e. $t=-\frac{\delta}{\gamma}$, the wavefunction becomes infinite, but this is precisely the singularity associated with the explosion.

\section{Conclusions}

We have investigated the maximal kinematical invariance group of general fluid mechanics and have found that it is a semi-direct product of the form $S L(2, R) \wedge G$ where $G$ is the static Galilei group. The incompressibility condition is not preserved by the above transformations and hence the viscosity terms transform covariantly only if the viscosity field $\zeta$ is constant in space (although not in time). In this case the fluid dynamic equations remain invariant if the viscosity fields transform like tensors

of rank $n$, where $n$ is the space dimension. The Navier-Stokes assumption of constant viscosity breaks the $S L(2, R)$ part of $\mathcal{G}$ to a two-parameter group of dilations and time translations in a tensorial manner.

The inversion transformation $\Sigma$ found in [4] is a special element of the $S L(2, R)$ and acts like the square root of parity. The transformations generated by the coset elements $g_{\Sigma}=\Sigma g$ act like the fourth roots of connected Galilei transformations. It is also pointed out that $\mathcal{G}$ is the Niederer group which is the maximal invariance group of the free Schrödinger equation. The reason for this is that $\mathcal{G}$ is actually the invariance group of the free Hamilton-Jacobi function, which is common to both fluid dynamics and the classical limit of the Schrödinger equation. It is surprising that such a basic result does not seem to be more widely known!

\section{Appendix}

In this appendix we show that the linear inhomogeneous transformations of the field variables considered in (18) are indeed the most general field transformations allowed. In order to show this we begin by noting that the most general transformations can be 
written as follows:

$$
\tilde{\phi} \equiv \tilde{\phi}(\xi, \tau, \phi) \quad \text { and } \quad \tilde{\rho} \equiv \tilde{\rho}(\xi, \tau, \rho)
$$

where $\tilde{\phi}$ and $\tilde{\rho}$ are a priori arbitrary functions of the old fields $\phi$ and $\rho$. Note that we are not allowing the fields to mix, but this is reasonable since $\phi$ and $\rho$ are really not on the same footing; whereas $\phi$ appears in the Action (17) only through its derivatives, $\rho$ appears without any derivatives. Using the fact that $x, t \rightarrow \xi, \tau$, the Hamilton-Jacobi function can be rewritten as follows:

$$
\frac{\partial \phi}{\partial t}-\frac{1}{2}\left(\frac{\partial \phi}{\partial x}\right)^{2}=\left(\frac{\partial \xi}{\partial t} \frac{\partial \phi}{\partial \xi}+\frac{\partial \tau}{\partial t} \frac{\partial \phi}{\partial \tau}\right)-\frac{1}{2}\left(\frac{\partial \xi}{\partial x} \frac{\partial \phi}{\partial \xi}+\frac{\partial \tau}{\partial x} \frac{\partial \phi}{\partial \tau}\right)^{2}
$$

Now it follows from

$$
\tilde{\phi} \equiv \tilde{\phi}(\xi, \tau, \phi) \Rightarrow \phi \equiv F(\xi, \tau, \tilde{\phi})
$$

that

$$
\frac{\partial \phi}{\partial \xi}=\frac{\partial F}{\partial \xi}+\frac{\partial \tilde{\phi}}{\partial \xi} \frac{\partial F}{\partial \tilde{\phi}} \quad \text { and } \quad \frac{\partial \phi}{\partial \tau}=\frac{\partial F}{\partial \tau}+\frac{\partial \tilde{\phi}}{\partial \tau} \frac{\partial F}{\partial \tilde{\phi}}
$$

We would now like to fix the form of $F$ by requiring the covariance of the HamiltonJacobi function i.e.

$$
\frac{\partial \phi}{\partial t}-\frac{1}{2}\left(\frac{\partial \phi}{\partial x}\right)^{2} \propto \frac{\partial \tilde{\phi}}{\partial \tau}-\frac{1}{2}\left(\frac{\partial \tilde{\phi}}{\partial \xi}\right)^{2}
$$

Substituting $(A 4)$ on the right hand side of $(A 2)$, we notice that we get terms of the form $(\partial \tilde{\phi} / \partial \tau)^{2}$ unless $\partial \tau / \partial x=0 \Rightarrow \tau \equiv \tau(t)$. Equating the coefficients of $\partial \tilde{\phi} / \partial \tau$ and $-(1 / 2)(\partial \tilde{\phi} / \partial \xi)^{2}$ because of covariance, we get

$$
\frac{\partial F}{\partial \tilde{\phi}}=\frac{\frac{\partial \tau}{\partial t}}{\left(\frac{\partial \xi}{\partial x}\right)^{2}}
$$

Requiring the coefficient of the $\partial \tilde{\phi} / \partial \xi$ term to vanish produces

$$
\frac{\partial F}{\partial \xi}=\frac{\frac{\partial \xi}{\partial t}}{\left(\frac{\partial \xi}{\partial x}\right)^{2}}
$$


Similarly requiring the coefficient of the $\tilde{\phi}$-independent terms to vanish we get

$$
\frac{\partial \xi}{\partial t} \frac{\partial F}{\partial \xi}+\frac{\partial \tau}{\partial t} \frac{\partial F}{\partial \tau}-\frac{1}{2}\left(\frac{\partial \xi}{\partial x}\right)^{2}\left(\frac{\partial F}{\partial \xi}\right)^{2}
$$

Substituting this equation in $(A 6)$ gives, after some algebra,

$$
\frac{\partial F}{\partial \tau}=-\frac{1}{2} \frac{\left(\frac{\partial \xi}{\partial t}\right)^{2}}{\left(\frac{\partial \xi}{\partial x}\right)^{2} \frac{\partial \tau}{\partial t}}
$$

From $(A 5)$ it follows that since $\tilde{\phi}$ is arbitrary, the left hand side changes whereas the right hand side, for a given $\xi, \tau, x, t$, is a constant in the field. Hence

$$
\frac{\partial F}{\partial \tilde{\phi}} \equiv s^{-1}(\xi, \tau)
$$

where $s^{-1}$ is a constant in the field variable. Solving the above differential equation we get

$$
F=s^{-1} \tilde{\phi}+\tilde{\lambda}(\xi, \tau) \Rightarrow \tilde{\phi}=s(\xi, \tau) \phi+\lambda(\xi, \tau)
$$

where $\lambda \equiv-s \tilde{\lambda}$. Further by taking a derivative of $(A 9)$ with respect to $\xi$ we see that

$$
\frac{\partial F}{\partial \xi}=\frac{\partial s^{-1}}{\partial \xi} \tilde{\phi}+s^{-1} \frac{\partial \tilde{\phi}}{\partial \xi}+\frac{\partial \tilde{\lambda}}{\partial \xi}
$$

Again from (A6) it is clear that the left hand side of the above equation is independent of $\tilde{\phi}$ and hence, $s^{-1}$ is independent of $\xi$. Similarly, by appealing to $(A 7)$ we can show that it is independent of $\tau$. Hence $s$ is a constant. A similar analysis may be carried out to establish the generality of the $\rho$ transformations in (18).

\section{Acknowledgements}

We thank Luke Drury for introducing us to this topic and for many useful discussions, David Saakian for raising the question of viscosity, O. Jahn for his interest, and R. Jackiw for valuable correspondence. 


\section{References}

1. Supernova Hydrodynamics Up Close: Science and Technology Review, Jan'/Feb' 2000; http://www.llnl.gov/str

2. I. Hachisu et al, Astrophysical Journal, 368, (1991), L27.

3. H. Sakagami and K. Nishihara, Physics of Fluids B 2, (1990), 2715.

4. L. O'C Drury and J. T. Mendonça, Physics of Plasmas 7, (2000) 5148.

5. S. Weinberg, Gravitation and Cosmology; John Wiley and Sons Inc. (1972).

6. E. C. G. Sudarshan and N. Mukunda, Classical Dynamics: A Modern Perspective, John Wiley \& Sons (1974).

7. For related work in this matter see, M. Hassaine and P. A. Horvathy, Ann. of Phys. 282 (2000) 218; Phys. Lett A279 (2001) 215; A. M. Grundland and L. Lalague, Can. J. Phys. 72, (1994) 362, Can. J. Phys. 73, (1995) 463; R. Jackiw, physics/0010042.

8. U. Niederer, Helvetica Physica Acta, 45 (1972) 802; C. R. Hagen Phys. Rev. D5 (1972) 377; R. Jackiw, Phys. Today 25 (1972) 23.

9. L. D. Landau and E. M. Lifshitz, Fluid Mechanics, Pergamon Press (1959).

10. A. Clebsch, Journal für die reine und angewandte Mathematik, 56 (1859), 1; H. Lamb, Hydrodynamics, Cambridge University Press, 1942. For related work, see S. Deser, R. Jackiw and A. P. Polychronakos, physics/0006056; R. Jackiw, V. P. Nair and So-Young Pi, hep-th/0004084. 